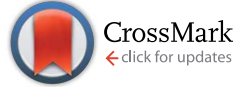

Cite this: RSC Adv., 2016, 6, 27047

Received 3rd January 2016 Accepted 27th February 2016

DOI: $10.1039 / c 6 r a 00100 a$

www.rsc.org/advances

\section{Biological fluorination from the sea: discovery of a SAM-dependent nucleophilic fluorinating enzyme from the marine-derived bacterium Streptomyces xinghaiensis NRRL B24674†}

\author{
Long Ma, ${ }^{\text {abc }}$ Yufeng Li, ${ }^{\text {ab }}$ Lingpei Meng, ${ }^{\text {ab }}$ Hai Deng, ${ }^{d}$ Yuyin Li, ${ }^{\text {ab }}$ Qiang Zhang ${ }^{e}$ \\ and Aipo Diao ${ }^{\text {ab }}$
}

\begin{abstract}
We have discovered and characterised a fluorinating enzyme from marine-derived Streptomyces xinghaiensis. It is able to install a fluorine atom into S-adenosyl-L-methionine (SAM) to form $5^{\prime}$-fluorodeoxy adenosine. To our knowledge, this is the first ever fluorinase unveiled from a marine source. It is 'the most efficient' fluorinase by far and, impressively, highly robust. Therefore it promises to be useful in different cases such as bio-transformation and synthetic biology.
\end{abstract}

Nature excites us by his hands to create a number of chemically complex structures with distinct bio-activities. It has been documented that over $70 \%$ of antibiotics entering clinical trials have been based on natural products. ${ }^{1}$ Marine organisms represent approximately half of the earth's biodiversity. Up until now, amongst $\sim 5000$ reported halogenated natural products, ${ }^{2}$ only six fluorinated products have been securely verified. This is very disproportionate to the abundance of fluorine in the earth, in despite of the fact that most fluorine elements are found in the earth crust and in insoluble forms. Importantly, fluorination of a molecule, especially a pharmaceutical, is of particular interest as halogenation is well known for its ability to tune the biophysical and bioactive properties to enhance drug efficacy through a range of mechanisms. ${ }^{3}$

In 2002, the first ever naturally occurring fluorinating enzyme (encoded by a flA gene) was isolated from Streptomyces cattleya, a terrestrial bacterium. It has been proven to have

${ }^{a}$ Key Laboratory of Industrial Fermentation Microbiology, Ministry of Education, School of Biotechnology, Tianjin University of Science \& Technology, Tianjin 300457, China. E-mail: malong@tust.edu.cn; Fax: +86 22 60602298; Tel: +86 22 60602948

${ }^{b}$ Tianjin Key Laboratory of Industry Microbiology, School of Biotechnology, Tianjin University of Science \& Technology, Tianjin 300457, China. Tel: +861334467254

${ }^{c}$ Biomolecular Sciences Research Complex, EaStCHEM School of Chemistry, University of St Andrews, Fife KY16 9ST, UK. E-mail:1m228@st.andrews.ac.uk

${ }^{d}$ Marine Biodiscovery Centre, Department of Chemistry, University of Aberdeen, Meston Walk, Aberdeen AB24 3UE, UK

${ }^{e}$ Tianjin 3rd Center Hospital, Tianjin 300170, China

$\dagger$ Electronic supplementary information (ESI) available. See DOI: 10.1039/c6ra00100a a unique ability to utilise inorganic fluorine ions and $S$-adenosyl-L-methionine (SAM) 1 to catalyse a $\mathrm{S}_{\mathrm{N}} 2$ biological nucleophilic reaction to generate $5^{\prime}$-fluorodeoxy adenosine ( $5^{\prime}$-FDA) 2 and L-methionine. ${ }^{4}$ It is the first committed enzyme in the fluorometabolites biosynthetic pathway Streptomyces cattleya. Another four enzymatic reactions are needed in turn to accomplish the biosynthesis of the final products of fluoroacetate (FAc) 6 and 4-fluorothreonine (4-FT) 7 in this organism. Fig. 1 describes this fluorometabolite biosynthetic pathway in detail. Taking one step further, in 2015, our work resulted in the discovery of a novel organofluorine natural product (2R3S4S)-5-fluoro-2,3,4-trihydroxypentanoic acid (5FHPA) 10 from a Ghanaian Streptomyces isolate named Streptomyces sp. MA37. This also led to the elucidation of a new fdr gene cluster, encoding a branched pathway for biosynthesis of 5-FHPA 10 from a intermediate 5-fluoro-D-ribose (5-FDR) 8, as shown in Fig. 1. ${ }^{5}$ This disclosure deepens the understanding of de novo fluorinated product biosynthesis. 5-FHPA 10 is the first secure identification of a new fluorinated natural product since 1998.

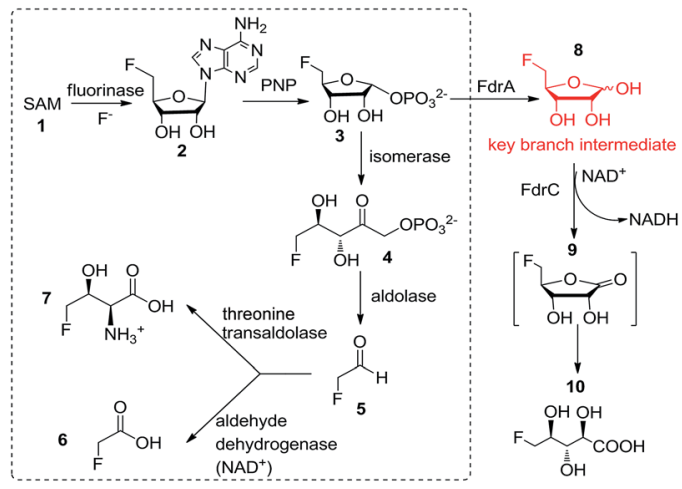

Fig. 1 Known microbial biosynthetic pathways of fluorinated natural products so far. The one in the dotted line box denotes the biosynthetic pathway to FAc and 4-FT established in Streptomyces cattleya. The one outside the box depicts the branched fluorometabolite pathway in Streptomyces sp. MA37. 


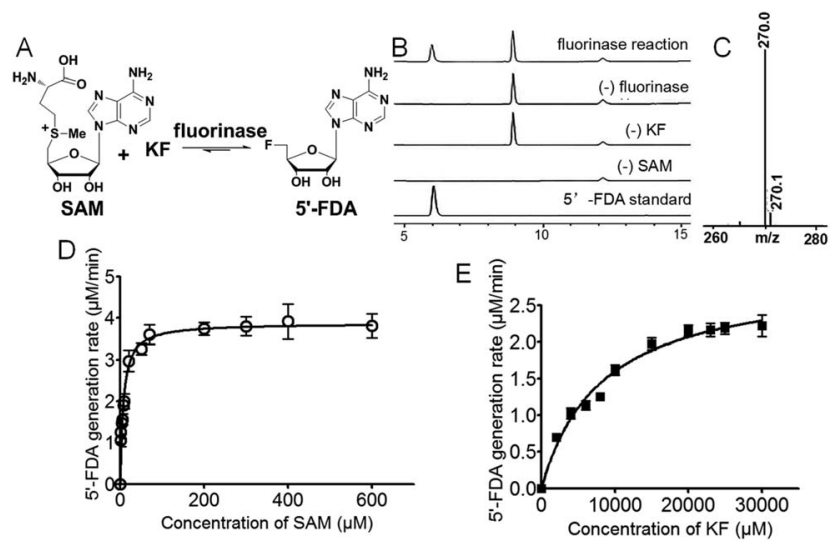

Fig. 2 Identification of a fluorinase from Streptomyces xinghaiensis, which is able to catalyse the conversion of fluoride and SAM dual substrates to generate $5^{\prime}$-FDA. (A) The chemical reaction catalysed by fluorinase. (B) HPLC analysis of the reaction denoted in (A). (C) LC-MS profile of the enzymatic reaction product $5^{\prime}$-FDA. The observed value $\left(\mathrm{m} / \mathrm{z}[\mathrm{M}+\mathrm{H}]^{+}=270.0\right)$ is consistent with the calculated value $(\mathrm{m} / \mathrm{z}$ $\left.[\mathrm{M}+\mathrm{H}]^{+}=270.10\right)$. Error bars are standard deviations for experiments performed in triplicate.

The fluorinase from $S$. cattleya stands for a unique resource for fluorination in biology. This fluorinase gene remained a sole representative for roughly a decade. Nevertheless, in 2014 our work revealed three other putative fluorinase genes that appeared in the genomes database of sequenced microorganisms (Streptomyces sp. MA37, Actinoplanes sp. N902-109 and Norcardia brasiliensis). PCR amplification of these genes and in vitro tests have confirmed they are all functional fluorinases. ${ }^{6}$

The known fluorinases are all FAD (flavin adenine dinucleotide)-, heme-, vanadium- or iron-independent, unlike some other reported halogenases. ${ }^{7}$ They are reminiscent of two functionally related genes: the duf-62 family $(\sim 30 \%$ in homology) and chlorinase ( $\sim 40 \%$ in homology). The abovementioned three types of enzymes catalyse closely related reactions with SAM as substrates, as shown in Fig. S1. $\dagger$ The gene alignment, phylogenetic study of known fluorianses, duf-62 and chlorinase is shown in Fig. $\mathrm{S} 2$ and $\mathrm{S} 3 \uparrow$ respectively. A unique characteristic of fluorinases to chlorinase and duf-62 enzymes is a 22 amino acid loop, which is deemed as a specific diagnostic signature. Chlorinase is from the marine organism Salinospora tropica, which performs related transformations on SAM but with a nucleophile chlorine other than a fluoride ion. ${ }^{8}$ It is tasked to be the first enzyme in a biosynthetic pathway, leading to production of a potent anti-cancer agent salinosporamide A. ${ }^{9}$ There are over 200 kinds of duf-62 in available genomes, mostly found in extremophile organisms. ${ }^{10}$ They have been shown to catalyse a reaction between SAM and a hydroxide ion to generate adenosine using a different mechanism from fluorinase. ${ }^{11}$

Streptomyces xinghaiensis NRRL B-24674 was isolated in 2009 from a marine sediment sample at Xinghai Bay, Dalian, China. This strain produces a novel alkaloid named xinghaiamine A. It has been genome sequenced in 2011. ${ }^{12}$ Our previous work has shown that fermentation of Streptomyces xinghaiensis NRRL B24674 resulted in production of FAc 6 probed by ${ }^{19} \mathrm{~F}-\mathrm{NMR}$. This indicates an active fluorinase gene in vivo, which can also be implied by in silico gene annotation. ${ }^{13}$ In this work, we reported one putative fluorinase from this bacterial strain. We have deduced the open reading frame of it and found that it has an $\sim 84 \%$ identity to the original $S$. cattleya fluorinase. A recombinant gene vector for over-expression was constructed, followed by protein induction and purification (Fig. S4 and S5 $\dagger$ ). We performed a HPLC and LC-MS survey to prove that it is a functional fluorinase, as shown in Fig. 2A-C. A kinetic study of this $S$. xinghaiensis fluorinase has been carried out and fitted with Michaelis-Menten curves (Fig. 2D and E).

In order to do so, we established a standard curve for $5^{\prime}$-FDA quantification based on HPLC measurements (Fig. S6 $\dagger$ ). The $K_{\mathrm{m}}$ with regard to SAM is $7.04 \pm 0.94 \mu \mathrm{M}$; that to $\mathrm{KF}$ is rather large, reaching $8.20 \pm 0.96 \mathrm{mM}$. It is highly agreeable with previously reported fluorinases, for which are all 'slow' and the $K_{\mathrm{m}}$ for KF is at the millimolar level. ${ }^{14}$ The $V_{\max }$ is $3.88 \pm 0.11 \mu \mathrm{M} \mathrm{min}{ }^{-1}$, in terms of SAM. The turnover number $\left(k_{\text {cat }}\right)$ was $0.277 \pm 0.007$ $\mathrm{min}^{-1}$, and the specificity constant is equal to $39.5 \pm 1.51 \mathrm{mM}^{-1}$ $\min ^{-1}$. We also performed a side-by-side comparison of $S$. xinghaiensis fluorinase with all other known fluorinases which is shown in Table 1 and Fig. S10.† From it we can see that the original $S$. cattleya enzyme is the least efficient, while the $S$. xinghaiensis fluorinase is the most efficient one, as evidenced by the fact that its specificity constant is around one order of magnitude larger than the rest. It has been previously reported that in the presence of L-amino acid oxidase (L-AAO), $S$. cattleya fluorinase is capable of utilising halogen ions $\mathrm{Cl}^{-}$to form $5^{\prime}$ chloro- $5^{\prime}$-deoxyadenosine ( $5^{\prime}$-CIDA). ${ }^{15}$ We have observed that $S$. xinghaiensis fluorinase is able to perform a reaction of the same kind. But this must require the presence of L-AAO to shift the

Table 1 The comparative enzyme kinetic data of fluorinases from different resources. Error bars are standard deviations for experiments performed in triplicate

\begin{tabular}{lllll} 
Fluorinase source & $V_{\max }\left(\mu \mathrm{M} \mathrm{min} \mathrm{min}^{-1}\right)$ & $\mathrm{SAM} K_{\mathrm{m}}(\mu \mathrm{M})$ & $k_{\text {cat }}=V_{\max } / C\left(\mathrm{~min}^{-1}\right)$ & $\begin{array}{l}\text { Specificity constant }= \\
k_{\mathrm{cat}} / K_{\mathrm{m}}\left(\mathrm{mM}^{-1} \mathrm{~min}^{-1}\right)\end{array}$ \\
\hline Streptomyces xinghaiensis & $3.88 \pm 0.11$ & $7.04 \pm 0.94$ & $0.277 \pm 0.007$ & $39.5 \pm 1.51$ \\
Streptomyces cattleya & $1.17 \pm 0.06$ & $29.4 \pm 5.80$ & $0.084 \pm 0.005$ & $2.84 \pm 0.14$ \\
Streptomyces sp. MA37 & $3.63 \pm 0.13$ & $86.0 \pm 11.3$ & $0.260 \pm 0.004$ & $3.02 \pm 0.19$ \\
Nocardia brasiliensis & $1.77 \pm 0.07$ & $30.4 \pm 4.24$ & $0.128 \pm 0.004$ & $4.22 \pm 0.42$ \\
Actinoplanes sp. N902-109 & $2.78 \pm 0.15$ & $43.1 \pm 7.99$ & $0.197 \pm 0.003$ & $4.58 \pm 0.50$
\end{tabular}




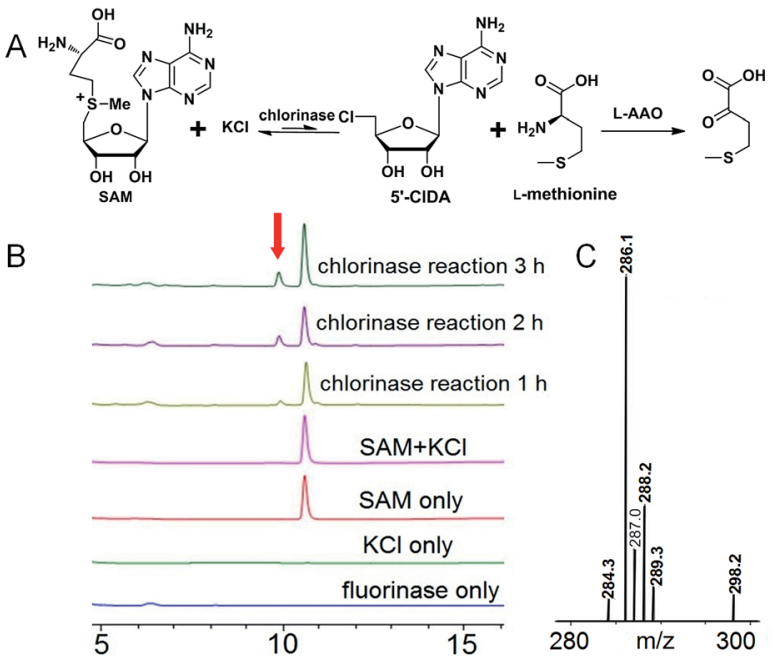

Fig. 3 Fluorinase from Streptomyces xinghaiensis NRRL B24674 is also a chlorinase, which is able to catalyse conversion of chloride and SAM to $5^{\prime}$-CIDA and L-methionine in the presence of L-amino acid oxidase. (A) The chemical reaction catalysed by fluorinase. (B) HPLC analysis of the reaction denoted in (A), the red arrow indicates $5^{\prime}$-CIDA production. (C) LC-MS profile of the enzymatic reaction product $5^{\prime}$ CIDA. The observed values $\left(m / z[M+H]{ }^{+35} \mathrm{Cl}=286.1,{ }^{37} \mathrm{Cl}=288.2\right)$ are consistent with the calculated values $\left(m / z[\mathrm{M}+\mathrm{H}]{ }^{+35} \mathrm{Cl}=286.07\right.$, $\left.{ }^{37} \mathrm{Cl}=288.07\right)$.

equilibrium of the reaction towards the organochlorine product (Fig. 3).

Next, we conducted a mutational study to find out the catalytically important amino acid residues (primers used are listed in Table S1†). Error bars are standard deviations for experiments performed in triplicate.

Fig. 4 shows mutational studies in a modeled $S$. xinghaiensis fluorinase structure. All the mutants showed some distortions in terms of secondary structure in comparison to the wild type enzyme; however, not to a great extent. This can be empirically reflected by far-UV circular dichroism (CD) spectroscopy recorded in the range of 190 to $260 \mathrm{~nm}$ (Fig. S7 $\dagger$ ). It has been proposed that $\mathrm{S} 158$ is a key residue for hydrogen bonding with fluorine ions. This can be mirrored by the current experiment, in which S158A only retains less than $20 \%$ activity compared with the WT one. D16 is crucial for substrate SAM binding and the F156 residue provides the rear of the hydrophobic pocket
A

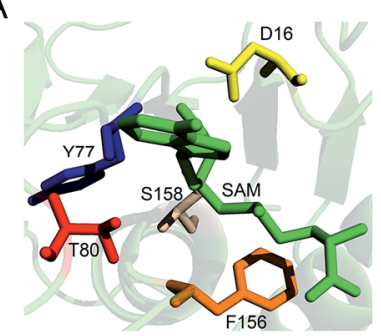

\section{$\mathrm{B}$}

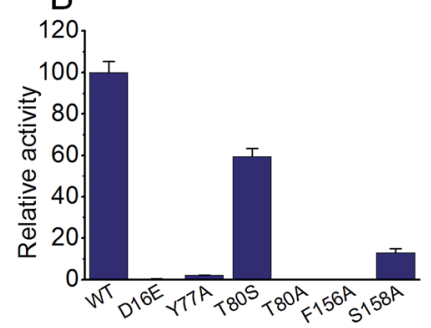

Fig. 4 Mutational studies of S. xinghaiensis fluorinase. (A) Mutation locations together with SAM. (B) The relative activities of all mutants. which is necessary to assist desolvation of fluoride ions. D16E and 156A mutants are detrimental to enzyme activity. T80A and Y77A severely abolish activity in our case, consistent with the mechanism that $\mathrm{T} 80$ and $\mathrm{Y} 77$ are able to facilitate halide binding. The mutated amino acids and relevant positions are shown in Fig. S8. $\dagger$

For this 'most efficient ever' S. xinghaiensis fluorianse, we would like to evaluate its stability and optimise its reaction conditions. Stability is an important property for all enzymes, particularly for those encountered in industrial processes. Optimal buffer ion and $\mathrm{pH}$ conditions always lead to great benefits including prolonging catalyst lifetime and increasing enzyme tolerance to external stress. ${ }^{16}$ Thermal shift analysis (TFA) is a quick and easy-to-use method for probing protein stability (mostly influenced by buffer compositions, metal ions, and $\mathrm{pH}$, etc.), solubility and protein-ligand interactions. It can be best performed using a conventional real-time PCR. Simply, when a protein undergoes thermal denaturation, its hydrophobic regions can bind the environmentally sensitive dye SYPRO Orange, which produces enhanced fluorescence. The fluorescence intensity correlates well with protein melting. ${ }^{17} \mathrm{We}$ did observe that ligands such as adenosine and the enzyme reaction product $5^{\prime}$-FDA enhance the protein stability and the wild-type enzyme is more stable than all mutants (Fig. S9 and Table S2 $\dagger$ ). We also tested different buffers and found Tris-HCl buffer ( $\mathrm{pH} 7.0$ ) is better for maintaining stability (Table S3†). We conducted a series of experiments to examine the feasibility of S. xinghaiensis fluorianse as a biocatalyst to catalyse $\mathrm{F}-\mathrm{C}$ bond formation. As shown in Fig. 5A, the optimal temperature for $S$. xinghaiensis fluorianse is around $40^{\circ} \mathrm{C}$ as expected. This is also a common temperature set for most enzymatic biotransformation reactions. The $\mathrm{pH}$ value seems crucial for its activity. Intriguingly, this enzyme was rather active in an acidic environment with a $\mathrm{pH}$ value as low as 4 . With a basic condition
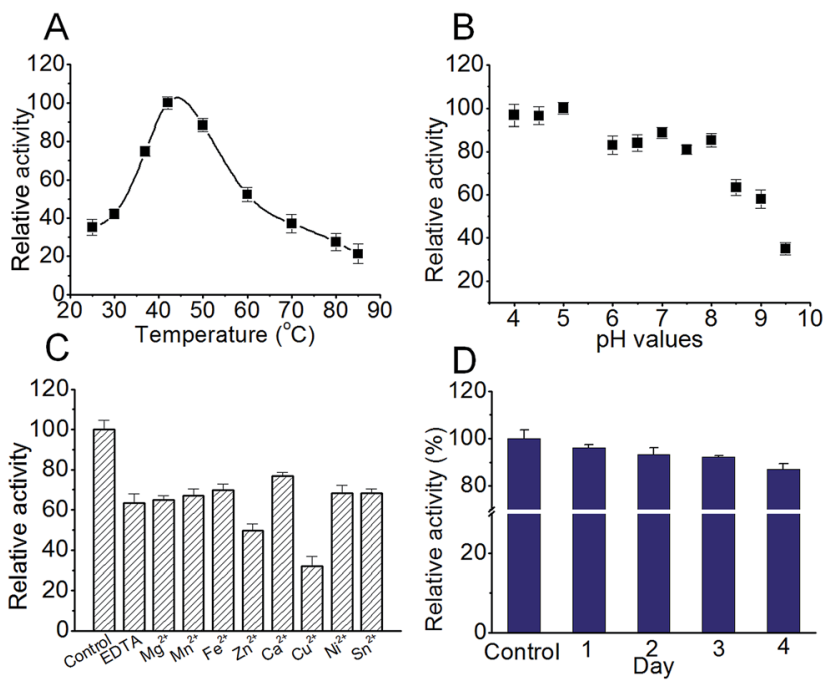

Fig. 5 Buffer composition, optimal $\mathrm{pH}$, temperature and ion condition for a fluorinating reaction catalysed by Streptomyces xinghaiensis fluorinase. Error bars are standard deviations for experiments performed in triplicate. 
(pH 8-9.5), its activity drops significantly (Fig. 5B). This observation renders the possibility of this enzyme to fulfil a biotransformation task in aqueous buffer with $\mathrm{pH}$ within the range from 4 to 8 . Fig. 5C shows that fluorination activity is highest in the 'control' condition (without EDTA and metal ions), and the addition of EDTA (to chelate metal ions) makes this enzyme considerably less active. This indicates that this fluorinase is possibly metal ion dependent. $\mathrm{Cu}^{2+}$ and $\mathrm{Zn}^{2+}$ can considerably inhibit activity. Furthermore, to be a candidate for industrial purposes, the enzyme should possess longevity in the buffer and be stable at ambient temperature. To test this, $S$. xinghaiensis fluorinase was placed at $25{ }^{\circ} \mathrm{C}$ in a $50 \mathrm{mM}$ Tris- $\mathrm{HCl}$ buffer ( $\mathrm{pH}$ 7.0). The enzyme activity was examined at different time points. Impressively, the enzyme showed excellent stability over 4 days, in which $\sim 87 \%$ of the activity remains in the 4 th day (Fig. 5D). Last, for a industrially relevant biocatalyst, a good expression platform is required. In the current study, we used $E$. coli to over-express this enzyme and have found that it is manageable to obtain $\sim 100 \mathrm{mg}$ of purified protein per gram of dried $E$. coli cells, which can be acquired from a 2 liter cell culture. Of course, other practical expression systems could be further developed for its production.

Naturally-derived products (small molecules, proteins and nucleic acids) have been recognised to be of high importance in sensing, diagnosis and treatment. ${ }^{18}$ Our report of this marineoriginated fluorinase, to our knowledge, is the first fluorinating enzyme discovered from a marine organism, which would expand the repertoire of exotic biological halogenation. It is also 'the most efficient' one. This might be because soluble fluoride ions are more prevalent in the sea than terrestrial sources, thus accelerating the evolution of fluorine biochemistry. We have also presented some fundamental data, which enable this enzyme to be more amenable for practical uses. In addition, theoretically and practically, halogenase, as biocatalysts can be used for generating halogenated natural product analogs with improved properties, which is highly industrially and bio-technologically relevant. ${ }^{19}$ In this work, we have afforded a most efficient fluorinase, which could be engineered or used as it is to fulfil bio-transformation purposes (e.g. fluorinated nucleic acid derivatives). The fluorinase gene can be used as a component in synthetic biology for generating the 'fluorinated version' of bio-active compounds using a microbial factory. ${ }^{20}$ Surely, further studies of this enzyme (gene) will be explored both experimentally and computationally. ${ }^{21}$

\section{Acknowledgements}

We thank Prof. David O'Hagan and Dr Qingzhi Zhang (University of St Andrews, UK) for their helpful discussion and for providing the synthetic $5^{\prime}$-FDA sample. This work is supported by National Natural Science Foundation of China (No. 81503086), a starting funding (No. 20140520) from Tianjin University of Science \& Technology, a research funding of "1000 Talents Plan" of Tianjin (to LM) and Foundation of Key Laboratory of Industrial Fermentation Microbiology of Ministry of Education and Tianjin Key Lab of Industrial Microbiology (No. 2015IM106).

\section{Notes and references}

1 D. J. Newman and G. M. Cragg, J. Nat. Prod., 2012, 75, 311335.

2 G. Gribble, Mar. Drugs, 2015, 13, 4044-4136.

3 (a) K. Müller, C. Faeh and F. Diederich, Science, 2007, 317, 1881-1886; (b) C. D. Murphy and G. Sandford, Expert Opin. Drug Metab. Toxicol., 2015, 11, 589-599; (c) Y. Lu, Y. Liu, Z. Xu, H. Li, H. Liu and W. Zhu, Expert Opin. Drug Discovery, 2012, 7, 375-383; (d) C. Odar, M. Winkler and B. Wiltschi, Biotechnol. J., 2015, 10, 427-446; (e) L. Ma and J. Liu, J. Ethnopharmacol., 2014, 158, 358-363.

4 (a) D. O'Hagan, C. Schaffrath, S. L. Cobb, J. T. G. Hamilton and C. D. Murphy, Nature, 2002, 416, 279; (b) C. Dong, F. Huang, H. Deng, C. Schaffrath, J. B. Spencer, D. O'Hagan and J. H. Naismith, Nature, 2004, 427, 561-565. 5 (a) L. Ma, A. Bartholome, M. H. Tong, Z. Qin, Y. Yu, T. Shepherd, K. Kyeremeh, H. Deng and D. O'Hagan, Chem. Sci., 2015, 6, 1414-1419; (b) Y. Wang, Z. Deng and X. Qu, F1000Research, 2014, 61, 1.

6 H. Deng, L. Ma, N. Bandaranayaka, Z. Qin, G. Mann, K. Kyeremeh, Y. Yu, T. Shepherd, J. H. Naismith and D. O'Hagan, ChemBioChem, 2014, 15, 364-368.

7 (a) J. L. R. Anderson and S. K. Chapman, Mol. BioSyst., 2006, 2, 350-357; (b) A. Butler and M. Sandy, Nature, 2009, 460, 848-854; (c) H. M. Senn, Front. Chem., 2014, 2, 98.

8 A. S. Eustaquio, F. Pojer, J. P. Noel and B. S. Moore, Nat. Chem. Biol., 2008, 4, 69-74.

9 L. Ma and A. Diao, Adv. Anticancer Agents Med. Chem., 2015, 15, 298-306.

10 H. Deng, C. H. Botting, J. T. G. Hamilton, R. J. M. Russell and D. O’Hagan, Angew. Chem., Int. Ed., 2008, 47, 5357-5361.

11 H. Deng and D. O'Hagan, Curr. Opin. Chem. Biol., 2008, 12, 582-592.

12 (a) X. Q. Zhao, W. J. Li, W. C. Jiao, Y. Li, W. J. Yuan, Y. Q. Zhang, H. P. Klenk, J. W. Suh and F. W. Bai, Int. J. Syst. Evol. Microbiol., 2009, 59, 2870-2874; (b) X. Zhao and T. Yang, J. Bacteriol., 2011, 193, 5543; (c) W. Jiao, F. Zhang, X. Zhao, J. Hu and J.-W. Suh, PLoS One, 2013, 8, e75994.

13 S. Huang, L. Ma, M. H. Tong, Y. Yu, D. O'Hagan and H. Deng, Org. Biomol. Chem., 2014, 12, 4828-4831.

14 X. Zhu, D. A. Robinson, A. R. McEwan, D. O'Hagan and J. H. Naismith, J. Am. Chem. Soc., 2007, 129, 14597-14604.

15 H. Deng, S. L. Cobb, A. R. McEwan, R. P. McGlinchey, J. H. Naismith, D. O'Hagan, D. A. Robinson and J. B. Spencer, Angew. Chem., Int. Ed., 2006, 45, 759-762.

16 (a) C. B. Poor, M. C. Andorfer and J. C. Lewis, ChemBioChem, 2014, 15, 1286-1289; (b) L. D. Finger, N. Patel, A. Beddows, L. Ma, J. C. Exell, E. Jardine, A. C. Jones and J. A. Grasby, Nucleic Acids Res., 2013, 41, 9839-9847; (c) L. Ma, X. Wu, G. G. Wilson, A. C. Jones and D. T. F. Dryden, Biochem. Biophys. Res. Commun., 2014, 449, 120-125.

17 (a) F. H. Niesen, H. Berglund and M. Vedadi, Nat. Protoc., 2007, 2, 2212-2221; (b) K. Huynh and C. L. Partch, in Curr. Protoc. Protein Sci., John Wiley \& Sons, Inc., 2001, 79:28.9.1-28.9.14. 
18 (a) L. Ma and S. L. Cockroft, ChemBioChem, 2010, 11, 25-34; (b) C. P. Wilson, C. Boglio, L. Ma, S. L. Cockroft and S. J. Webb, Chem.-Eur. J., 2011, 17, 3465-3473; (c) L. Ma, K. Chen, D. J. Clarke, C. P. Nortcliffe, G. G. Wilson, J. M. Edwardson, A. J. Morton, A. C. Jones and D. T. F. Dryden, Nucleic Acids Res., 2013, 41, 4999-5009; (d) L. Ma and A. Diao, Chem. Commun., 2015, 51, 1023310235; (e) L. Ma, G. Wu, Y. Li, P. Qin, L. Meng, H. Liu, Y. Li and A. Diao, Nanoscale, 2015, 7, 18044-18048; $(f)$ T. Sabir, A. Toulmin, L. Ma, A. C. Jones, P. McGlynn, G. F. Schroder and S. W. Magennis, J. Am. Chem. Soc., 2012, 134, 6280-6285.

19 (a) D. R. M. Smith, S. Gruschow and R. J. M. Goss, Curr. Opin. Chem. Biol., 2013, 17, 276-283; (b) M. C. Walker and
M. C. Y. Chang, Chem. Soc. Rev., 2014, 43, 6527-6536; (c) S. A. Shepherd, C. Karthikeyan, J. Latham, A.-W. Struck, M. L. Thompson, B. R. K. Menon, M. Q. Styles, D. Leys and J. Micklefield, Chem. Sci., 2015, 6, 3454-3460; (d) S. Brown and S. E. O'Connor, ChemBioChem, 2015; (e) J. FernándezLucas, Appl. Microbiol. Biotechnol., 2015, 99, 4615-4627.

20 (a) A. S. Eusthquio, D. O'Hagan and B. S. Moore, J. Nat. Prod., 2010, 73, 378-382; (b) M. C. Walker and M. C. Y. Chang, Chem. Soc. Rev., 2014, 43, 6527-6536.

21 (a) L. C. Blasiak and C. L. Drennan, Acc. Chem. Res., 2009, 42, 147-155; (b) A. Timmins and S. P. de Visser, Advances in Protein Chemistry and Structural Biology, Academic Press, 2015, vol. 100, pp. 113-151; (c) H. M. Senn, D. O'Hagan and W. Thiel, J. Am. Chem. Soc., 2005, 127, 13643-13655. 